Monatsschr Kinderheilkd 2021 · 169:200 https://doi.org/10.1007/s00112-020-01110-8 Angenommen: 14. Dezember 2020

Online publiziert: 5. Januar 2021

(c) Springer Medizin Verlag GmbH, ein Teil von Springer Nature 2021

\section{Redaktion}

R. Kerbl, Leoben

G. Krandick, Oberhaching

T. Niehues, Krefeld

P. Voitl, Wien

\section{Originalpublikation}

Sass L, Vinding RK, Stokholm J et al (2020) High-dose vitamin D supplementation in pregnancy and neurodevelopment in childhood. A prespecified secondary analysis of a randomized clinical trial. JAMA Network Open 3(12):e2026018. https://doi.org/10. 1001/jamanetworkopen.2020.26018

Studie. Die vorliegende Arbeit ist eine vorab geplante Zweitauswertung der „Copenhagen Prospective Studies on Asthma in Childhood". Von 623 schwangeren Frauen wurden 315 in die Hochdosisgruppe randomisiert und erhielten ab der 24. Schwangerschaftswoche (SSW) bis eine Woche nach der Entbindung $2400 \mathrm{IE}$ Vitamin $\mathrm{D}_{3} /$ Tag zusätzlich zu der Standarddosis von 400 IE/Tag. 308 Frauen erhielten in diesem Zeitraum nur die Standarddosis. 277 Kinder aus der Hochdosis- und 274 aus der Normaldosisgruppe konnten bis zum Alter von 6 Jahren nachverfolgt werden. Ausgeschlossen worden waren Mütter mit endokrinen, kardialen oder renalen Vorerkrankungen, ebenso wie Kinder mit einem Gestationsalter $<37$ SSW, einem Geburtsgewicht $<2500 \mathrm{~g}$ oder mit neurologischen Erkrankungen. Eine Woche nach der Entbindung lagen die Vitamin-D-Spiegel im Mittel bei 43,18 vs. $28,94 \mathrm{ng} / \mathrm{ml}$.

Primärer Endpunkt der Studie war die kognitive Entwicklung, die im Alter von 2,5 Jahren mithilfe der Bayley-IIISkalen durch ausgebildete Untersucher beurteilt wurde. Weitere entwicklungsneurologische Parameter wurden von den Eltern erfasst und waren das Errei-

Guido Krandick

Oberhaching, Deutschland

\title{
Hochdosiertes Vitamin D in der Schwangerschaft ohne Effekt auf entwicklungsneurologische Parameter des Kindes
}

chen motorischer Meilensteine (DenverTest und WHO-Kriterien), die Sprachentwicklung (MacArthur-Bates Communicative Development Inventories), die generelle Entwicklung (Ages and Stages Questionnaire) sowie emotionelle und Verhaltensprobleme im Alter von 6 Jahren (Strengths and Difficulties Questionnaire). Die Studie war doppelblind, bis das jüngste Kind 3 Jahre alt war, und untersucherverblindet bei allen weiteren Kontakten.

Ergebnisse. Im Ergebnis gab es keine Unterschiede zwischen Vitamin-DHochdosis- und -Normaldosisgruppe in Bezug auf Bayley-Skalen (Score-Differenz 0,34 [95\%-Konfidenzintervall (KI) $-1,32-1,99] ; p=0,70)$, motorische Meilensteine ( $\beta=0,08$ [95\%-KI -0,26-0,43]; $p=0,64)$, generelle Entwicklung (Median [Interquartilenabstand (IQA)] 50 [50-55] vs. 50 [50-55]; $p=0,62)$ und emotionelle und Verhaltensprobleme („odds ratio“ 0,76 [95\%KI 0,53-1,09]; $p=0,14)$. In der Sprachentwicklung gab es mit einem Jahr keine Unterschiede bezüglich der Wortproduktion (Median [IQA] 2 [0-6] vs. 3 [1-6]), jedoch mit 2 Jahren eine verminderte Wortproduktion in der Hochdosisgruppe (Median [IQA] 232 [113-346] vs. 253 [149-382,5], $p=0,02)$. Nach Studienplanung wurde auf eine Bonferroni-Korrektur für multiple Testung zunächst verzichtet. Wurde diese jedoch durchgeführt, verschwand dieser Unterschied.

\section{Kommentar}

Die Ergebnisse dieser randomisierten, kontrollierten Studie entsprechen denen aus der Mehrzahl vorhergehender Beobachtungsstudien. Es ist also nunmehr belegt, dass die Standarddosis von 400IE/Tag Vitamin $\mathrm{D}_{3}$ in einer dänischen Population für ein gesundes entwicklungsneurologisches Outcome der Kinder adäquat ist.

\section{Korrespondenzadresse}

\section{Dr. Guido Krandick}

Tisinstraße 56a, 82041 Oberhaching, Deutschland

mail@dr-krandick.de

Interessenkonflikt. G. Krandick gibt an, dass kein Interessenkonflikt besteht. 